\title{
Association between Nonalcoholic Fatty Liver Disease (NAFLD) and Coronary Artery Disease (CAD) in Patients with Angina Pectoris
}

\author{
Atoosa Adibi ${ }^{1}$, Majid Jaberzadeh-Ansari ${ }^{1 *}$, Amir-Reza Dalili ${ }^{1}$, Navid Omidifar ${ }^{2}$, Masoumeh Sadeghi ${ }^{1,3}$ \\ ${ }^{1}$ Department of Radiology, School of Medicine, Isfahan University of Medical Sciences, Isfahan, Iran \\ ${ }^{2}$ Department of Pathology, Shiraz University of Medical Sciences, Shiraz, Iran \\ ${ }^{3}$ Cardiac Rehabilitation Research Center, Cardiovascular Research Center, Isfahan University of Medical Sciences, Isfahan, Iran \\ Email: *majidjansari@yahoo.com
}

Received July 20, 2013; revised August 16, 2013; accepted August 30, 2013

Copyright (C) 2013 Atoosa Adibi et al. This is an open access article distributed under the Creative Commons Attribution License, which permits unrestricted use, distribution, and reproduction in any medium, provided the original work is properly cited.

\begin{abstract}
Background: Prevalence of nonalcoholic fatty liver disease (NAFLD) is increasing in Iranian population. The aim of this study was the investigation of association between nonalcoholic fatty liver disease (NAFLD) and coronary artery disease (CAD) in patients with angina pectoris. Methods: 82 patients with confirmed CAD by coronary angiography and 82 individuals with normal coronary angiography, as the control group, were selected. Hepatic ultrasound scanning was performed in all the subjects to determinate the NAFLD. Data were analyzed by SPSS software and independent T-test and Chi-square tests. Results: Findings gathered from the patients with angina pectoris showed that the prevalence of NAFLD in CAD patients was higher than the control group. Grade of fatty liver and the portal vein size in CAD patients were significantly more than the control group. Conclusion: NAFLD is a risk factor of CAD in the angina pectoris patients. Increase in the prevalence of NAFLD can lead to the increase in cardiovascular diseases.
\end{abstract}

Keywords: Non-Alcoholic Fatty Liver Disease; Coronary Artery Disease; Angina Pectoris

\section{Introduction}

Nonalcoholic fatty liver disease (NAFLD) is one of the most common hepatic disorders [1]. Prevalence of this disorder is $10 \%-30 \%$ in general population and its prevalence is increasing in both developing and developed countries [2-5]. NAFLD has been strongly associated with insulin resistance and metabolic syndrome; most of patients are overweight or obese, and they have glucose metabolism deficiencies, dyslipidemia, and hypertension which all are the risk factors of NAFLD [6-8].

Coronary artery disease (CAD) is the first cause of death in rich countries and the second cause of death in the medium and low income countries [9]. Risk factors of CAD include age, gender [10-14], diabetes mellitus [15-18], low physical activity, smoking, hyperlipidemia, metabolic syndrome, and diet [9]. Study that was conducted in Iranian population showed that $24 \%$ of adults in Isfahan city had these risk factors [19].

NAFLD has been thought to be a new risk factor for

\footnotetext{
${ }^{*}$ Corresponding author.
}

CAD. Other new risk factors are inflammatory markers and fibrinolytic and hemostatic dysfunctions. Recent studies revealed that NAFLD patients had an accelerated risk of increased carotid intima and media thickness [20,21], reduced endothelial function [22], increased coronary artery calcification [23,24], and increased arterial stiffness [20]. According to some studies, NAFLD and CAD have the similar risk factors including dyslipidemia, hypertension, physical inactivity, insulin resistance and inflammation, therefore, a possible relationship between NAFLD and CAD can be assumed and evaluated [8-25]. In addition, increase in liver enzymes has been identified as an independent risk factor for CAD and a large number of patients with CAD had abnormal liver function tests [26].

As regards, in Iran, the most common cause of increase in liver enzymes is NAFLD, and the increase in liver enzymes has been demonstrated as a risk factor for CAD. Aim of this study was investigation of the possible association between NAFLD and CAD in patients with angina pectoris. 


\section{Methods and Materials}

\subsection{Participants}

This is a case-control study conducted on the patients with angina pectoris and the candidates for coronary angiography. Patients were chosen from those who referred to the Chamran and Khorshid hospitals in Isfhan city. Patients with the history of recent myocardial infarction or other general disorders, pregnant women and alcoholics were excluded. Coronary angiography was performed and the severity of CAD was assessed in those with the positive coronary angiography results. 82 patients with the confirmed CAD in coronary angiography and 82 individuals with matched age, sex, sugar and lipid profile, and weight and height as the control group with the normal coronary angiography results were selected with simple randomization for the present study. Sample size was calculated by this equation [27]:

$$
\begin{gathered}
n=\frac{\left(z_{1-\alpha / 2}+z_{1-\beta}\right)^{2}\left[P_{1}\left(1-P_{1}\right)+P_{2}\left(1-P_{2}\right)\right]}{d^{2}} \\
z_{1-\alpha / 2}=1.96, z_{1-\beta}=0.84, P_{1}=0.1, P_{2}=0.01
\end{gathered}
$$

The relevant ethics committee of Isfahan University of Medical Sciences granted approval for this research (project number: 385,316). Informed consent form was completed by the subjects.

\subsection{Conventional X-Ray Coronary Angiography}

All participants were undergone standard clinical diagnostic x-ray angiography. Conventional x-ray coronary angiography was performed with an integrated digital cardiac catheter imaging system. All of the coronary angiographies were performed by one investigator. Then, the $x$-ray angiograms were investigated by the three cardiologists, and, the percentage of stenosis diameter, as the reference diameter, was determined, according to the SI Unit.

\subsection{Hepatic Ultrasound Scanning}

Hepatic ultrasound scanning was performed in all the subjects by one trained radiologist, who was unaware of all the clinical and laboratory characteristics of the patients, using a Toshiba Nemio 30 scanner (just vision model, Toshiba CO. Ltd., Tokyo, Japan) with a $3.5 \mathrm{MHz}$ linear transducer. Participants were asked to lie in the supine and $60^{\circ}$ left lateral positions during quiet inspiration. The presence or absence and the grading of fatty infiltration of the liver were recorded. Absence or grade 0 of fatty infiltration was considered to be the normal liver echogenicity. Grade 1 (mild) of fatty infiltration was considered for the slightly increased liver echogenicity, with normal visualization of the diaphragm and the intrahepatic vessel borders. Grade 2 (moderate) of fatty infiltration was defined when the echogenicity of liver moderately increased, with slightly impaired visualization of the diaphragm or intrahepatic vessels. Grade 3 (severe) of fatty infiltration was considered when the liver echogenicity markedly increased with the poor visualization of diaphragm, the intrahepatic vessels and the posterior portion of the right liver lobe [28].

\subsection{Statistical Analysis}

All data were analyzed by SPSS (version 16) software. Data are presented as the mean \pm standard deviation (SD) or frequencies. Statistical analyses included the independent T-test (for continues variables) and the Chisquare test (for categorical variables). p $<0.05$ was considered statistically significant.

\section{Results}

This study was conducted on 82 CAD patients and 82 normal participants. Case and control groups were matched by age, sex, blood sugar and lipid profile, weight and height. There was 24 females (29.26\%) and 58 males (70.73\%) in each group (CAD patients and control group). Basic characteristics of participants in two groups are shown in Table 1.

Our results showed that the patients with CAD had significantly higher NAFLD compared to the control group $(p=0.001)$. In addition, the proportions of subjects with NAFLD in CAD patients group were significantly higher than control group in the two genders. 50 males and 20 females in CAD group had NAFLD (Table 2).

The mean severity of NAFLD in CAD group was significantly higher than the control group (1.24 vs 0.59 ). Frequencies of various grades of NAFLD in the CAD patients and control group are shown in Figure 1.

Table 1. Basic characteristics of participants.

\begin{tabular}{cccc}
\hline & Normal group & CAD patients group & $\mathrm{p}$ \\
\hline Age (year) & $44.2 \pm 4.54$ & $44.8 \pm 4.20$ & 0.83 \\
Weight (kg) & $72.8 \pm 1.48$ & $73.4 \pm 2.30$ & 0.63 \\
Height (cm) & $163.8 \pm 2.77$ & $164.8 \pm 4.08$ & 0.66 \\
FBS (mg/dl) & $115.8 \pm 10.91$ & $117.8 \pm 11.47$ & 0.78 \\
TC (mg/dl) & $211.6 \pm 7.43$ & $215.5 \pm 7.04$ & 0.45 \\
TG (mg/dl) & $160.2 \pm 8.07$ & $166.25 \pm 6.29$ & 0.26 \\
HDL (mg/dl) & $44.8 \pm 3.27$ & $43.0 \pm 2.58$ & 0.40 \\
LDL (mg/dl) & $108.0 \pm 8.09$ & $115.4 \pm 1.67$ & 0.08 \\
\hline
\end{tabular}

Mean \pm standard deviation. CAD = coronary artery disease, FBS = fasting blood sugar, $\mathrm{TC}=$ total cholesterol, $\mathrm{TG}=$ triglyceride, $\mathrm{HDL}=$ high density lipoproteins, LDL = low density lipoproteins. 


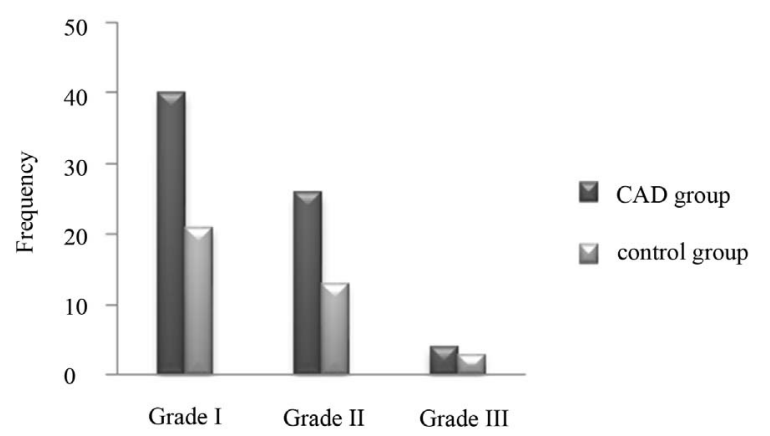

Figure 1. Frequencies of various grades of NAFLD in CAD patients and control group.

Table 2. Presence of NAFLD and other relevant variables among the two groups of study.

\begin{tabular}{cccc}
\hline & Normal group & $\begin{array}{c}\text { CAD patients } \\
\text { group }\end{array}$ & $\mathrm{p}$ \\
\hline NAFLD (\%), total & $45.12 \%$ & $85.36 \%$ & 0.001 \\
NAFLD (\%), men & $32.76 \%$ & $86.2 \%$ & $\mathrm{p}<0.05$ \\
NAFLD (\%), women & $50 \%$ & $83.3 \%$ & $\mathrm{p}<0.05$ \\
Hepatomegaly (\%) & $3.7 \%$ & $4.9 \%$ & 0.7 \\
Grade of fatty liver & $0.59 \pm 0.30$ & $1.24 \pm 0.25$ & $\mathrm{p}<0.001$ \\
spleen size (mm) & $98.6 \pm 4$ & $97.8 \pm 4.75$ & 0.8 \\
Portal vein size (mm) & $9.45 \pm 0.33$ & $9.97 \pm 0.38$ & $\mathrm{p}<0.05$ \\
\hline
\end{tabular}

Mean \pm standard deviation. NAFLD $=$ nonalcoholic fatty liver disease, $C A D$ $=$ coronary artery disease.

There was no significant difference between the CAD patients and control group, about the liver size (4.9\% vs $3.7 \%)$. The mean size of spleen in the control group was higher than the CAD group; however, this difference was not statistically significant. The mean size of portal vein in the CAD patients was significantly greater than the control group (Table 2).

\section{Discussion}

The present study shows that the prevalence of NAFLD in CAD patients with angina pectoris was more than the control group in the whole population and the two genders. Grade of fatty liver and the portal vein size in CAD patients were more than the control group in the whole population and the two genders. Grade of fatty liver and the portal vein size in CAD patients were more than the control group. Therefore, NAFLD can be a risk factor and the cause of CAD in patients with angina pectoris. Previous studies shows that NAFLD independently enhances the risk of CAD and peripheral vascular diseases [23-27,28]. Gastaldelli et al. reported that fatty liver is significantly associated with the increased CAD risk and reduced insulin sensitivity in non-diabetic subjects [29].
According to the findings, cardiovascular mortality rate is increasing in NAFLD patients [30]. Targher et al. [31] reported that NAFLD is a predictor of cardiovascular disease CVD in type II diabetes. Hamaguchi et al. [32] showed the relationship between NAFLD and CVD in healthy patients. Several mechanisms were suggested for the relationship between NAFLD and CAD. Oxidative stress increases in NAFLD and finally leads to the modification of low-density lipoproteins (LDL) and produces oxidized LDL that is a risk factor for atherosclerosis and CAD [33]. Studies showed that NAFLD is strongly associated with insulin resistance (IR) as well as reduced insulin sensitivity [34]. IR leads to the endothelial dysfunction and finally atherosclerosis [35]. Studies show that the levels of interleukin 6 (IL-6) and C-reactive protein (CRP) are increased in NAFLD, and NAFLD was considered as a chronic inflammatory condition. Inflammation mediates all the stages of atherosclerosis [9]. In addition, patients with NAFLD have reduced levels of adiponectin [36], and lower plasma adiponectin concentration is associated with early CAD onset and multiple atherosclerotic lesions in coronary arteries.

The limitation of our study is that NAFLD was diagnosed based on the ultrasonography appearance of liver. Gold standard for the diagnosis of NAFLD is liver biopsy but as this process is invasive and costly, we did not perform liver biopsy.

\section{Conclusion}

This study was conducted among the patients with angina pectoris showed that the NAFLD was more prevalent in CAD patients. Thus, NAFLD is a risk factor for the presence of CAD in patients with angina pectoris and clinicians must pay attention to this risk factor in health care.

\section{REFERENCES}

[1] G. C. Farrell and C. Z. Larter, "Nonalcoholic Fatty Liver Disease: From Steatosis to Cirrhosis," Hepatology, Vol. 43, No. 2, 2006, pp. 99-112. doi:10.1002/hep.20973

[2] J. D. Browning, L. S. Szczepaniak, R. Dobbins, P. Nuremberg, J. D. Horton, J. C. Cohen, et al., "Prevalence of Hepatic Steatosis in an Urban Population in the United States: Impact of Ethnicity,” Hepatology, Vol. 40, No. 6, 2004, pp. 1387-1395. doi:10.1002/hep.20466

[3] J. A. Welsh, S. Karpen and M. B. Vos, "Increasing Prevalence of Nonalcoholic Fatty Liver Disease among United States Adolescents, 1988-1994 to 2007-2010,” Journal of Pediatrics, Vol. 162, No. 3, 2013, pp. 496-500. doi:10.1016/j.jpeds.2012.08.043

[4] G. Bedogn, L. Miglioli, F. Masutti, C. Tiribelli, G. Marchesini and S. Bellentani, "Prevalence of and Risk Factors for Nonalcoholic Fatty Liver Disease: The Dionysos Nutrition and Liver Study," Hepatology, Vol. 42, No. 1, 
2005, pp. 44-52. doi:10.1002/hep.20734

[5] X. Hu, Y. Huang, Z. Bao, Y. Wang, D. Shi, F. Liu, et al., "Prevalence and Factors Associated with Nonalcoholic Fatty Liver Disease in Shanghai Work-Units,” BMC Gastroenterology, Vol. 12, No. 1, 2012, p. 123. doi:10.1186/1471-230X-12-123

[6] X. Ying, Y. Jiang, Y. Qian, Z. Jiang, Z. Song and C. Zhao, "Association between Insulin Resistance, Metabolic Syndrome and Nonalcoholic Fatty Liver Disease in Chinese Adults," Iranian Journal of Public Health, Vol. 41, No. 1, 2012, pp. 45-49.

[7] G. Musso, R. Gambino, S. Bo, B. Uberti, G. Biroli, G. Pagano, et al., "Should nonalcoholic Fatty Liver Disease Be Included in the Definition of Metabolic Syndrome? A Cross-Sectional Comparison with Adult Treatment Panel III Criteria in Nonobese Nondiabetic Subjects,” Diabetes Care, Vol. 31, No. 3, 2008, pp. 562-568. doi:10.2337/dc07-1526

[8] M. Hamaguchi, N. Takeda, T. Kojima, A. Ohbora, T. Kato, H. Sarui, et al., "Identification of Individuals with Non-Alcoholic Fatty Liver Disease by the Diagnostic Criteria for the Metabolic Syndrome," World Journal of Gastroenterology, Vol. 18, No. 13, 2012, pp. 1508-1516. doi:10.3748/wjg.v18.i13.1508

[9] W. Nseir, A. Shalata, A. Marmor and N. Assy, "Mechanisms Linking Nonalcoholic Fatty Liver Disease with Coronary Artery Disease," Digestive Diseases and Sciences, Vol. 56, No. 12, 2011, pp. 3439-3449. doi:10.1007/s10620-011-1767-y

[10] C. Ruhl and J. Everhart, "Epidemiology of Nonalcoholic Fatty Liver,” Clinical Liver Disease, Vol. 8, No. 3, 2004, pp. 501-519. doi:10.1016/j.cld.2004.04.008

[11] L. Carulli, A. Lonardo, S. Lombardini, G. Marchesini and P. Loria, "Gender, Fatty Liver and GGT," Hepatology, Vol. 44, No. 1, 2006, pp. 278-279. doi:10.1002/hep. 21218

[12] B. Neal, S. MacMahon and N. Chapman, "Effects of ACE Inhibitors, Calcium Antagonists, and Other Blood Pressure-Lowering Drugs,” Lancet, Vol. 356, No. 9246, 2000, pp. 1955-1964. doi:10.1016/S0140-6736(00)03307-9

[13] G. Donati, B. Stagni, F. Piscaglia, N. Venturoli, A. M. Morselli-Labate, L. Rasciti, et al., "Increased Prevalence of Fatty Liver in Arterial Hypertensive Patients with Normal Liver Enzymes: Role of Insulin Resistance," Gut, 2004, Vol. 53, No. 7, pp. 1020-1023. doi:10.1136/gut.2003.027086

[14] S. Yokohama, M. Yoneda, M. Haneda, S. Okamoto, M. Okada, K. Aso, et al., "Therapeutic Efficacy of an Angiotensin II Receptor Antagonist in Patients with Nonalcoholicsteatohepatitis,” Hepatology, Vol. 40, No. 5, 2004, pp. 1222-1225. doi:10.1002/hep.20420

[15] J. Stamler, O. Vaccaro, J. Neaton and D. Wentworth, "Diabetes, Other Risk Factors, and 12-Yr Cardiovascular Mortality for Men Screened in the Multiple Risk Factor Intervention Trial,” Diabetes Care, Vol. 16, No. 2, 1993, pp. 434-444. doi:10.2337/diacare.16.2.434

[16] A. Adlerberth, A. Rosengren and L. Wilhelmsen, "Diabetes and Long-Term Risk of Mortality from Coronary and Other Causes in Middle-Aged Swedish Men. A General Population Study,” Diabetes Care, Vol. 21, No. 4, 1998, pp. 539-545. doi:10.2337/diacare.21.4.539

[17] R. de Marco, F. Locatelli, G. Zoppini, G. Verlato, E. Bonora and M. Muggeo, "Cause-Specific Mortality in Type 2 Diabetes. The Verona Diabetes Study,” Diabetes Care, Vol. 22, No. 5, 1999, pp. 756-761. doi:10.2337/diacare.22.5.756

[18] G. Marchesini, R. Marzocchi, F. Agostini and E. Bugianesim, "Nonalcoholic Fatty Liver Disease and Metabolic Syndrome," Current Opinion in Lipidology, Vol. 16, No. 4, 2005, pp. 421-427. doi:10.1097/01.mol.0000174153.53683.f2

[19] N. Sarraf-Zadgan, G. Sadri, H. Malek-Afzali, M. Baghaei, N. Mohammadi-Fard, S. Shahrokhi, et al., "Isfahan Healthy Heart Program: A Comprehensive Integrated Community-Based Program for Cardiovascular Disease Prevention and Control,” Acta Cardiologica, Vol. 58, No. 4, 2003, pp. 309-320. doi:10.2143/AC.58.4.2005288

[20] A. Brea, D. Mosquera, E. Martín, A. Arizti, J. L. Cordero and E. Ros, "Nonalcoholic Fatty Liver Disease Is Associated with Carotid Atherosclerosis: A Case-Control Study," Arteriosclerosis, Thrombosis, and Vascular Biology, Vol. 25, No. 5, 2005, pp. 1045-1050. doi:10.1161/01.ATV.0000160613.57985.18

[21] C. H. Tsai, T. C. Li and C. C. Lin, "Metabolic Syndrome as a Risk Factor for Nonalcoholic Fatty Liver Disease," Southern Medical Journal, Vol. 101, No. 9, 2008, pp. 900-905. doi:10.1097/SMJ.0b013e31817e8af9

[22] G. N. Ioannou, N. S. Weiss, E. J. Boyko, D. Mozaffarian and S. P. Lee, "Elevated Serum Alanine Aminotransferase Activity and Calculated Risk of Coronary Heart Disease in the United States,” Hepatology, Vol. 43, No. 5, 2006, pp. 1145-1151. doi:10.1002/hep.21171

[23] A. Ugur, T. Sedat, B. Serhat, T. Yusuf, K. Tarkan and C. Atiye, "Association between Nonalcoholic Fatty Liver Disease and Coronary Artery Disease," Coronary Artery Disease, Vol. 18, No. 6, 2007, pp. 433-436. doi:10.1097/MCA.0b013e3282583c0d

[24] L. Pacifico, V. Cantisani, P. Ricci, J. F. Osborn, E. Schiavo, C. Anania, et al., "Nonalcoholic Fatty Liver Disease and Carotid Atherosclerosis in Children," Pediatric Research, Vol. 63, No. 4, 2008, pp. 423-427. doi:10.1203/PDR.0b013e318165b8e7

[25] I. S. Okosun, K. M. Chandra, A. Boev, J. M. Boltri, S. T. Choi, D. C. Parish, et al., "Abdominal Adiposity in US Adults: Prevalence and Trends, 1960-2000," Preventive Medicine, Vol. 39, No. 1, 2004, pp. 197-206. doi:10.1016/j.ypmed.2004.01.023

[26] Y. Lin, H. Lo and J. Chen, "Sonographic Fatty Liver, Overweight and Ischemic Heart Disease,” World Journal of Gastroenterology, Vol. 11, No. 31, 2005, pp. 48384842.

[27] J. E. Bartlett, J. W. Kotrlik and C. C. Higgins, “Organizational Research: Determining Appropriate Sample Size for Survey Research,” Information Technology, Learning, and Performance Journal, Vol. 19, No. 1, 2001, pp. 4350 .

[28] S. Y. Choi, D. Kim, H. J. Kim, J. H. Kang, S. J. Chung, 
M. J. Park, et al., "The Relation between Non-Alcoholic Fatty Liver Disease and the Risk of Coronary Heartdisease in Koreans," The American Journal of Gastroenterology, Vol. 104, No. 8, 2009, pp. 1953-1960. doi:10.1038/ajg.2009.238

[29] G. Targher, F. Marra and G. Marchesini, "Increased Risk of Cardiovascular Disease in Non-Alcoholic Fatty Liver Disease: Causal Effect or Epiphenomenon?” Diabetologia, Vol. 51, No. 11, 2008, pp. 1947-1953. doi:10.1007/s00125-008-1135-4

[30] A. Gastaldelli, M. Kozakova, K. Hojlund, A. Flyvbjerg, A. Favuzzi, A. Mitrakou, et al., "Fatty Liver Is Associated with Insulin Resistance, Risk of Coronary Heart Disease, and Early Atherosclerosis in a Large European Population,” Hepatology, Vol. 49, No. 5, 2009, pp. 15371544. doi:10.1002/hep.22845

[31] C. Matteoni, Z. Younossi, T. Gramlich, N. Boparai, Y. Liu and A. McCullough, "Nonalcoholic Fatty Liver Disease: A Spectrum of Clinical and Pathological Severity," Gastroenterology, Vol. 116, No. 6, 1999, pp. 1413-1419. doi:10.1016/S0016-5085(99)70506-8

[32] G. Targher, L. Bertolini, R. Padovani, F. Poli, L. Scala, L. Zenari, et al., "Non-Alcoholic Fatty Liver Disease Is As- sociated with Carotid Artery Wall Thickness in DietControlled Type 2 Diabetic Patients,” Journal of Endocrinological Investigation, Vol. 29, No. 1, 2006, pp. 5560.

[33] M. Hamaguchi, T. Kojima, N. Takeda, C. Nagata, J. Takeda, H. Sarui, et al., "Nonalcoholic Fatty Liver Disease Is a Novel Predictor of Cardiovascular Disease," World Journal of Gastroenterology, Vol. 13, No. 10, 2007, pp. 1579-1584.

[34] M. Ahmed and C. Byrne, "Obstructive Sleep Apnea Syndrome and Fatty Liver: Association or Causal Link?” World Journal of Gastroenterology, Vol. 16, No. 34, 2010, pp. 4243-4252. doi:10.3748/wjg.v16.i34.4243

[35] E. Bugianesi, A. McCullough and G. Marchesini, "Insulin Resistance: A Metabolic Pathway to Chronic Liver Disease," Hepatology, Vol. 42, No. 5, 2005, pp. 987-1000. doi:10.1002/hep.20920

[36] G. Targher, L. Bertolini, R. Padovani, S. Rodella, G. Zoppini, L. Zenari, et al., "Relations between Carotid Artery Wall Thickness and Liver Histology in Subjects with Nonalcoholic Fatty Liver Disease,” Diabetes Care, Vol. 29, No. 6, 2006, pp. 1325-1330. doi:10.2337/dc06-0135 